\title{
Adoption des normes IFRS dans l'espace OHADA : Fondement théorique, intérêt et enjeux
}

\section{Adoption of IFRS standards in the OHADA space: Theoretical basis, interest and challenges}

\author{
Nyasseu Emma Joachim, (Doctorant) \\ Faculté des sciences économique et de gestion \\ Université de Dschang, Cameroun \\ Yepdo Florentin Gaël, (Doctorant) \\ Faculté des sciences économique et de gestion \\ Université de Dschang, Cameroun
}

\author{
Tatang Atabonfack Pulchérie Léonie, (Doctorante) \\ Faculté des sciences économique et de gestion \\ Université de Dschang, Cameroun
}

\author{
Cheguem Shupo Davine Myskinole, (Doctorante) \\ Faculté des sciences économique et de gestion \\ Université de Dschang, Cameroun
}

\begin{abstract}
Résumé : Du fait des changements profonds qu'apportent les nouveaux concepts sur lesquels se base le référentiel de l'IASB, le passage aux normes IFRS a été qualifié d'une révolution comptable. Le but d'harmonisation de cette institution se réalise à travers la diffusion mondiale progressive des normes IFRS. Toutefois, les entreprises de la zone OHADA sont concernées par ce changement. Compte tenu de la rareté des travaux de recherche dans le domaine, nous essayons d'analyser à travers ce papier, l'adoption de telles normes en contexte OHADA. Pour y parvenir, cet article rappelle le mécanisme de
\end{abstract}


passage aux normes IFRS dans l'espace OHADA, étudie ensuite l'intérêt pour les entreprises de cette zone et enfin met en évidence les enjeux liés à un tel projet. Les principaux résultats montrent que loin d'être un simple outil de présentation et de communication de l'information financière, les IFRS constituent pour les entreprises de la zone OHADA, un levier d'attraction des investisseurs et d'intégration économique.

Mots clés : normes IFRS, OHADA, révolution comptable, référentiel.

\begin{abstract}
Due to the profound changes brought about by the new concepts on which the IASB framework is based, the transition to IFRS standards has been described as an accounting revolution. The goal of harmonizing this institution is achieved through the gradual worldwide dissemination of IFRS standards. However, companies in the OHADA zone are affected by this change. Given the scarcity of research work in the field, we try to analyze through this paper, the adoption of such standards in the OHADA context. To achieve this, this article recalls the mechanism for transitioning to IFRS in the OHADA area, then studies the interest for companies in this area and finally highlights the issues associated with such a project. The main results show that far from being a simple tool for the presentation and communication of financial information, the IFRS constitute for companies in the OHADA zone, a lever of investor attraction and economic integration.
\end{abstract}

Keywords: IFRS, OHADA, accounting revolution, repository.

\title{
1. Introduction
}

La vulgarisation mondiale des marchés des capitaux et l'expansion des sociétés multinationales constituent la semence qui a fait germer la nécessité pour les entreprises d'horizon divers d'adopter un langage commun des affaires. Le décloisonnement des marchés ainsi que les ententes économiques, monétaires et sociales qui en découlent, se répercutent jusqu'à sur le plan comptable. Ainsi, en vue de prendre les décisions d'investissement les plus fécondes, les agents économiques se réfèrent au système comptable qui constitue la base de toute la production de l'information économique. Dans cette optique, l'harmonisation des normes comptables a constitué une visée incontournable, particulièrement au sein de l'Union Européenne. L'objectif étant de mettre à la disposition des utilisateurs, une information accessible, pertinente et reconnue.

Afin d'éviter d'être en marge des évolutions internationales qui, aujourd'hui sont irréversibles et seules les limites restent inconnues, le normalisateur OHADA a ainsi rendu obligatoire l'usage des normes IFRS par certaines entreprises. Par ailleurs, eu égard aux divergences des principes fondamentaux sur lesquels se basent le système comptable local et international, l'adoption d'un tel référentiel dans le contexte OHADA n'est pas sans apporter quelques difficultés. En réalité, la transition aux normes IFRS suppose la remise en question d'approches comptables traditionnelles au profit de nouveaux concepts traduisant une véritable révolution comptable. 
Par conséquent, pour expliquer sa diffusion au niveau mondial et l'intérêt de son adoption par les entreprises de la zone OHADA, il nous parait judicieux de faire un rappel sur les mécanismes de l'avènement de ce nouveau système comptable international dans l'Union Européen et de mettre en lumière sa philosophie. Tout de même, l'analyse des principaux points de divergences entre le référentiel comptable OHADA et celui de l'IASB permet de conclure qu'à première vue, le référentiel comptable international parvient à fournir une traduction réelle et fidèle de la situation économique de toutes entreprises adoptive (Escafre, 2012). Toutefois, le passage aux normes IFRS par les entreprises de la zone OHADA représente des enjeux importants qu'il conviendrait de bien appréhender et implémenter dans ce processus de mise en œuvre.

\section{Avènement du système comptable international en Europe}

Fondamentalement, le phénomène d'harmonisation comptable international tire ses origines en Europe avant de toucher d'autres pays eu égard de l'accroissement des échanges et du décloisonnement des marchés. Ainsi, l'harmonisation européenne par voie de directive a montré ses limites du fait de multiples options que le traitement des comptes admet d'une part, mais également à cause de la lenteur de la procédure à travers la nécessité d'obtenir un fort consensus politique des Etats membres d'autre part (Haoudi K, 2015). C'est au regard de ces limites que l'Union Européen décide de soutenir formellement le projet d'harmonisation comptable international implémenté par l'IASC (International Accounting Standards Committee). La décision prise en 2000 par l'Europe suite à la proposition faite par la commission Européenne (CE) concernant l'adoption généralisée des normes IFRS par les sociétés cotées constitue un véritable tremplin. Dès lors, la grande obstination de voire appliquer un système comptable unique au sein de l'Union Européenne a motivée ce dernier à appuyer cette proposition et à la confirmer à travers un règlement du parlement et du conseil. L'objectif de ce règlement (CE N¹606/2002) du 19 juillet 2002 vise l'adoption et l'application des normes de l'IASB (International Accounting Standards Board) dans tous les pays membres pour les exercices ouvert à partir du $1^{\text {er janvier } 2005 .}$

L'adoption par l'Union Européenne des normes de l'IASB a suscité l'intérêt de plusieurs pays à travers le monde. Certains pays ont délibérément opté pour l'abandon total des normes nationales pour s'arrimer à ce nouveau système comptable. D'autres ont fait converger progressivement ou partiellement leur norme nationale vers celles de portée internationale. Ces faits témoignent à suffisance autant d'indicateurs sur la pertinence de ce référentiel par rapport aux systèmes comptables locaux et même par rapport aux normes US-GAAP ${ }^{1}$.

\footnotetext{
${ }^{1}$ Generally Accepted Accounting Principles (United States). Ce sont les normes comptables Américaines En français, ils sont dénommé Principes Comptables Généralement Admis
} 
Toutefois, l'adoption des normes IFRS par l'Europe constitue une réelle révolution du fait du changement fondamental du rôle assigné à la comptabilité. Cette révolution se matérialise par le passage de la primauté de la vision économique sur l'apparence juridique. En effet, les normes comptables internationales sont bâties sur des fondements théoriques statiques de la comptabilité et présentent des spécificités distinctes des autres normes qui ont une logique dynamique de la comptabilité (Richard, 2005).

\subsection{Définition et vision des normes comptables internationales IFRS}

Les IFRS (International Financial Reporting Standard) sont des normes comptables élaborées par le Bureau des standards comptables internationaux IASB (International Accounting Standards Board) donc le principal objectif vise à garantir une meilleure transparence des chiffres comptables via la production harmonisée, qui facilitera la compréhension et la comparaison au niveau mondial. C'est en 1965 que cette idée fut exprimée pour la première fois par Wilkinson sous le vocable « uniformité comptable » (Barbu, 2004). Cette idée traduisait l'établissement d'un seul jeu d'état financier pour tous les investisseurs d'horizon divers. Ces derniers sont selon le cadre conceptuel, considérés comme les destinataires privilégiés de ces états comptables.

\section{La philosophie générale du référentiel comptable IFRS}

Le référentiel doit permettre la compréhension et la comparabilité des comptes établis à partir de principes identiques pour répondre aux besoins des utilisateurs internes et externes. Il est déconnecté de tout environnement juridique et fiscal et se place au-dessus des spécificités nationales. Il est élaboré à partir d'une approche économique traduisant la réalité de l'activité économique de l'entreprise par rapport au marché avec une prééminence du fond sur la forme et une évaluation fréquente des opérations à la juste valeur. L'objectif est d'informer les utilisateurs des états financiers, principalement les investisseurs, afin qu'ils puissent prendre des décisions économiques en toute connaissance à partir :

- De la situation financière (bilan) ;

- De la performance (compte de résultat);

- Des variations de la situation financière (tableau des flux de trésorerie);

- Des éléments non comptabilisés dans le bilan et dans le compte de résultat (annexe);

- Des variations des capitaux propres (état des variations des capitaux propres).

Les normes IFRS apportent ainsi une nouvelle philosophie dans l'environnement comptable. Elles sont arrimées aux évolutions économiques et les informations financières communiquées selon ses 
formats reflètent la réalité économique de l'entreprise. De nos jours, plusieurs normes locales à l'instar des normes OHADA convergent vers elle.

\subsection{L'investisseur comme destinataire privilégié de l'information financière}

Le cadre conceptuel de l'IASB (support théorique de l'élaboration des normes), identifie les investisseurs et créanciers comme étant les utilisateurs privilégiés de l'information comptable et financière. Selon Cornier et al. (2007), l'objectif des états financiers est la production d'information utile aux investisseurs pour leurs prises de décision et l'allocation de leurs ressources. En effet, l'adoption d'un système comptable unique permettra aux actionnaires et investisseurs externes de faire une meilleure comparaison de la performance des différentes entreprises appartenant à un secteur d'activité qui leur est commun.

\subsection{La juste-valeur : pierre angulaire du référentiel international}

Etant donnée l'ampleur de l'impact de l'utilisation dans l'évaluation des entreprises, la juste-valeur constitue la pierre angulaire du référentiel international. Ainsi, il remplace le coût historique, de même que la substance économique l'emporte sur la forme juridique. La norme IAS 32 définie la juste-valeur comme «le montant par lequel un actif peut être échangé ou un passif émis entre deux parties volontaires et bien informées dans le cadre d'une transaction à intérêts contradictoires et effectué dans les conditions de concurrence normale ». Elle se présente de ce fait comme un principe permettant aux représentations comptables d'être plus encrées dans la réalité économique. Cependant, son application reste partielle et assez limitée et ne s'applique pas à tous les actifs et passifs de l'entreprise. selon Lenormand et al. (2012), la juste-valeur est souvent abusivement assimilée à une valeur de marché. Toutefois, l'existence de biens particuliers dont l'évaluation ne peut se faire sur un marché, appelles l'introduction de nouveaux outils permettant cette évaluation (Haoudi K, 2015). De ce fait, on passe donc d'une approche objective fondée sur les prix de marché (mark-to-market), à une approche subjective fondée sur des valeurs modélisées (mark-to-model).

\subsection{La prééminence de la réalité économique sur l'apparence juridique}

Selon ce principe, pour que l'information représente d'une manière pertinente les transactions et autres événements qu'elle vise à représenter, il est nécessaire qu'ils soient enregistrés et présentés en accord avec leur substance et la réalité économique et non pas seulement selon leur forme juridique. Ainsi, toutes ressource économique contrôlée par l'entreprise est à paraitre dans le bilan de cette dernière, même si elle n'en détient pas le titre de propriété. Toutefois, ce principe concerne également les biens que les entités contrôlées de fait, qui doivent faire l'objet d'une obligation de consolidation. Pour Disle et Noel (2007), ce passage d'une approche juridique et historique de la comptabilité à une approche 
économique, plus ancrée dans la réalité, constitue un autre argument utilisé pour défendre la thèse de la plus grande pertinence des données comptables établies selon le référentiel de l'IASB.

\section{Vulgarisation mondiale des IFRS}

Depuis la signature en 1973 de la charte de l'IASC dont l'objectif visait l'élaboration d'un référentiel comptable à même de réaliser la transparence et la comparabilité des entreprises d'horizon divers, de progrès significatifs ont été réalisés en matière de normalisations et d'harmonisations comptables internationales. Cependant, les efforts déployés par cette instance en vue de promouvoir l'utilisation des normes IAS au niveau mondial n'ont pas abouti au résultat espéré. En effet, ces progrès se sont matérialisés vers les années 2001 par la reforme de l'IASC devenu IASB et les nouvelles normes désormais appelées IFRS. Cet à partir de ce moment qu'un changement notable a eu lieu. Ainsi, les normes IFRS ont été retenue au sein de l'Union Européenne et application rendue obligatoire par les entreprises cotées de cet espace à partir de l'exercice ouvert en 2005.

L'évolution du cadre comptable devant prendre en compte les normes admises par l'ensemble de la communauté internationale, le but visé par l'harmonisation ne peut se limiter qu'au plan européen (Elatife, 2012). Avec la mondialisation des économies et le développement des marchés boursiers, les pays de l'espace OHADA comme toute économie en développement, ont besoin de concours financier extérieur pour amorcer véritablement leur développement (Waha, 2013). L'adoption des normes internationales par l'Union européenne fut un véritable catalyseur pour leur préférence par d'autres pays à travers le monde. Ainsi, Certains pays ont adopté purement et simplement les IFRS, d'autres on fait évoluer leur système comptable pour y intégrer les IFRS. Aux États-Unis, même les normalisateurs qui avaient montré par le passé une politique plutôt hostile vis-à-vis des normalisations étrangères, sont entrés depuis 2002 dans un projet commun de révision du cadre conceptuel de la comptabilité avec l'IASB. L'objectif de cette coopération est de faire converger à terme, les Generaly Accepted Accounting Principles (US-GAAP) et les IFRS. Cependant, les résultats d'une étude menée par Danjou (2013), révèlent que près de 120 pays au monde ont imposé ou permis l'application des normes IFRS. En mars 2018, la fondation IFRS a publiée sur son site internet le profil de 166 pays représentant 99\% du PIB mondial. Ainsi, 144 pays soit 87\% d'entre eux exigent l'utilisation des normes IFRS et 12 autres pays les autorisent.

De nos jours, presque tous les pays d'Amérique du Sud autorisent ou exigent les IFRS pour l'établissement des états financiers. En 2010, le Brésil a adopté les IFRS pour toutes les sociétés cotées et les banques. Le Chili l'a adopté pour toutes les sociétés ouvertes en 2012. De même, en 2012, l'Argentine a adopté les IFRS pour toutes les sociétés (sauf les banques et les sociétés d'assurance qui continuent de se conformer aux exigences locales). Au Mexique, les IFRS ont été adoptées pour toutes 
les entités cotées, à l'exception des banques et les sociétés d'assurance qui appliquent les normes mexicaines d'information financière. Par ailleurs, un projet de convergence est en cours afin de supprimer toutes les divergences entre ce référentiel et les IFRS. De même, ces normes sont exigées dans plusieurs autres pays d'Amérique latine et des Caraïbes. Au Canada, l'information financière a été marquée par une vague de changements important depuis l'adoption des IFRS en 2011 en tant que principes comptables généralement admis pour les entreprises ayant une obligation d'information du public et les entités commerciales du secteur public. Selon Finharmony (2012), treize pays africains soit $20,1 \%$ adoptent les IFRS partiellement ou entièrement, mais le constat fait est que ces pays sont logés dans les zones anglophones d'Afrique. Toutefois, ce constat justifie les liens qu'entretiennent ces pays avec la métropole.

\section{Révision comptable OHADA et adoption des normes IFRS}

La vulgarisation et la promotion des normes comptables internationales en Afrique s'imposent comme une nécessité dans un contexte économique mondial libéralisé, où seules les entreprises compétitives tirent leurs épingles du jeu. Toutefois, le système comptable OHADA qui a été adopté le 24 mars 2000 présente beaucoup de lacune et reste dans l'ombre au niveau international. Il est donc appelé à évoluer davantage afin de converger dans un avenir proche avec les normes IFRS qui sont celles de portée internationale. C'est dans cette mouvance que 1'OHADA a élaboré le 25 octobre 2012, un projet d'amélioration du climat des investissements (PACI) financé par la banque mondiale via la signature de la convention d'un don de 15 millions de dollars ${ }^{2}$. Le désir d'amélioration de l'information comptable et financière dans les sociétés était la composante principale de ce projet. C'est ainsi qu'une reforme de l'acte uniforme portant organisation et harmonisation des comptabilités des entreprises a été signée en janvier 2017, ceci dans le but d'adopter les meilleurs pratiques et standards internationaux dans ce domaine.

Réunis le 26 janvier 2017, lors de sa $43^{\text {ème }}$ session tenue à Brazzaville (Congo), le Conseil des Ministres de l'Organisation pour l'Harmonisation en Afrique du Droit des Affaires (OHADA) a adopté le nouvel Acte Uniforme de l'OHADA relatif au Droit Comptable et à l'Information Financière (AUDCIF). Cet Acte a pour but d'adapter les règles comptables et financières régissant les entreprises œuvrant dans l'espace OHADA aux évolutions de la normalisation comptable internationale, dans le respect du contexte juridique et économique des États parties. Dès lors, l'une des innovations majeure issue de cette révision est l'adoption des normes IFRS qui est rendue obligatoire pour les entreprises cotées et celles sollicitant des financements dans le cadre d'un appel public à l'épargne dès le $1^{\text {er }}$ janvier 2019 (Art. 113).

\footnotetext{
${ }^{2}$ Source $:$ http:/project.banque mondiale.org/procurement/notioeoverview ?
} 


\subsection{Avantages de l'adoption des normes IFRS pour l'espace OHADA}

La vulgarisation des IFRS dans le monde n'a pas laissé indifférent les pays de la zone OHADA. Dans l'optique de parler le même langage des affaires afin de sortir du chao que connaissent les entreprises de cet espace, le normalisateur OHADA a adopté les normes IFRS pour les entreprise cotées sur une bourse et celles désirant des financements dans le cadre d'un appel public à l'épargne. Ce dernier a de ce fait, le souci d'assister et d'accompagner les entreprises dans leur quête des marchés étrangers. Il étant ses actions dans plusieurs domaines au rang desquels celui des normes internationales. Ainsi, ces normes deviennent progressivement la clé de voûte de tous les systèmes globalisés et dans les organisations internationales qui régulent le processus de mondialisation (Dafir, 2013). Le rôle fondamental joué par la comptabilité dans ce nouveau contexte explique la volonté grandissante du normalisateur à instaurer les règles d'un système comptable de haute qualité répondant au mieux aux exigences des investisseurs et bailleurs de fonds.

Au-delà des raisons évoquées ci-dessus, l'adoption des normes IFRS dans l'espace OHADA pourrait émaner d'un autre facteur notamment moins contraignant. En effet, les entreprises de l'espace OHADA pourraient appliquer les normes IFRS par simple effet de mimétisme. Pour Barbu et Piot (2012), si tel est le cas, leur adoption n'est pas perçue comme un levier de performance économique, mais plutôt comme un moyen d'asseoir la légitimité de l'entreprise.

\subsection{Un choix guidé par une action isomorphique}

De multiples travaux menés dans le contexte européen ont mis en lumière l'existence d'autres facteurs pouvant motiver l'adoption des normes internationales. Du fait de l'absence de telles études dans le contexte de l'OHADA, nous ne pouvons extrapoler ces résultats sur le choix de l'adoption des normes IFRS par le normalisateur OHADA. Toutefois, nous pouvons poser des hypothèses sur la conformité de ces résultats par rapport au cas singulier de l'OHADA.

Dans la poursuite de l'analyse du processus d'homogénéisation des organisations, DiMaggio et Powell (1983), mettent en évidence l'existence d'isomorphismes. Selon ces auteurs, l'isomorphisme est un processus de contrainte qui force une unité appartenant à une population à ressembler aux autres qui sont confrontées aux mêmes conditions environnementales. Les processus d'isomorphismes contribuent à asseoir la légitimité des firmes (Démaria, 2008 ; Trabelsi, 2013).

$\checkmark$ L'isomorphisme prend la forme coercitive lorsque le changement est la conséquence de pressions produites par la force, la persuasion et la collusion (Touron, 2000). Les pressions émanent d'abord de l'État et conduisent alors les organisations à s'adapter à l'environnement légal, mais elles s'exercent aussi en dehors du cadre strictement légal (Anne le Manh, 2009). 
$\checkmark$ L'isomorphisme prend la forme mimétique lorsque les organisations imitent d'autres organisations considérées comme plus performantes (Barbu, 2006). L'incertitude à laquelle doivent faire face les organisations tient un rôle déterminant sur les choix, ainsi Mezias (1990) affirme que l'incitation à imiter les pratiques institutionnalisées sera plus forte pour les firmes faisant face à l'incertitude.

$\checkmark$ L'isomorphisme normatif prend sa source dans la professionnalisation définit comme «le combat des membres d'une occupation pour définir les conditions et les méthodes de leur travail pour contrôler la production des producteurs, établir une base cognitive et la légitimation de l'autonomie de leur activité » (Dimagio et Powell, 1983). Selon ces auteurs, la professionnalisation tient à deux éléments, la formation et l'existence de réseaux professionnels.

Dans une étude menée en 2008 en France dont le but visait à comprendre en profondeur les choix d'options comptables des groupe français, Démaria confirme qu'afin de répondre au contexte environnemental et institutionnel, les organisations adoptent un comportement isomorphique pour légitimer leurs choix. De même, en analysant les influences ressenties par les dirigeants d'entreprises françaises cotées du fait de l'adoption des normes internationales, Barbu et Piot (2012) confirment au travers des réponses obtenues, la rémanence du phénomène institutionnel dans des dites normes. En référence à la théorie néo-institutionnelle qui démontre le seuil de légitimité de l'entreprise, l'étude menée ébranle un isomorphisme coercitif notamment, souvent en coexistence avec un isomorphisme mimétique.

$\mathrm{Au}$ sein de l'espace OHADA, nous constatons de prime à bord que l'obligation d'adopter les IFRS pour les entreprises cotées émane d'un isomorphisme coercitif. Ainsi, ces entreprises croulent sous le poids des pressions formelles de l'Etat et des exigences des marchés boursiers. Toutefois, les multiples efforts déployés par le normalisateur OHADA en ce sens comme mentionné ci-dessus, témoigne d'une grande volonté à établir une base juste à l'ensemble des informations produites par les entreprises de cet espace.

\section{Principales divergences entre les normes OHADA et IAS/IFRS}

Au niveau international, les études menées par Price Waterhouse (1975) considèrent les différences comptables comme étant le résultat de différentes options adoptées dans divers pays pour le traitement des mêmes opérations. Ainsi, les normes IFRS s'affirment comme la référence pour les pays développés et ou émergents qui s'en inspirent largement, notamment dans le but ultime de suivre le vent de la mondialisation. Cependant, le système comptable OHADA révisé, bien qu'inspiré des normes comptables internationales, présente encore quelques points de divergences avec les IFRS. 
Tableau 1: comparaison entre les normes comptables SYSCOHADA révisé et IFRS (points de divergences)

\begin{tabular}{|c|c|c|}
\hline NORMES IAS/IFRS & SYSCOHADA Révisé & Observations \\
\hline $\begin{array}{l}\text { IAS } \mathbf{1 8} \\
\text { Les produits des activités } \\
\text { ordinaires }\end{array}$ & $\begin{array}{l}\text { Le référentiel n'a pas défini le concept de produits Ordinaires. } \\
\text { Contrairement aux IFRS, les produits avec différé de paiement ne } \\
\text { font pas l'objet d'une actualisation. } \\
\text { Contrairement aux IFRS, les escomptes accordés en cas de paiement } \\
\text { anticipé sont comptabilisés en charges financières. }\end{array}$ & Divergence \\
\hline $\begin{array}{l}\text { IAS } 20 \\
\text { Comptabilisation des subventions } \\
\text { publiques et informations à } \\
\text { fournir sur l'aide publique }\end{array}$ & $\begin{array}{l}\text { Selon le Système comptable OHADA, les subventions } \\
\text { d'investissements sont enregistrées en capitaux propres alors qu'elles } \\
\text { sont enregistrées en compte de tiers, produits différés en norme } \\
\text { IFRS. Par ailleurs, les subventions d'investissements ne viennent pas } \\
\text { en déduction du coût d'acquisition du bien dans le référentiel } \\
\text { OHADA (deuxième méthode préconisée par les IFRS). }\end{array}$ & Divergence \\
\hline $\begin{array}{l}\text { IAS 21 } \\
\text { Effets des variations des cours des } \\
\text { monnaies étrangères }\end{array}$ & $\begin{array}{l}\text { les écarts de conversion portant sur les éléments monétaires sont } \\
\text { comptabilisés dans les comptes de tiers contrairement aux normes } \\
\text { IFRS qui comptabilisent directement les pertes et gains latents en } \\
\text { résultat (pas donc de compte d'écart de conversion en compte de } \\
\text { tiers en IFRS) }\end{array}$ & Divergence \\
\hline $\begin{array}{c}\text { IAS 26 } \\
\text { Comptabilité et rapports } \\
\text { financiers des régimes de retraite }\end{array}$ & Norme non retenue par le SYSCOHADA & Divergence \\
\hline $\begin{array}{l}\text { IAS } 32 \\
\text { Instruments financiers } \\
\text { Présentation }\end{array}$ & $\begin{array}{l}\text { Contrairement aux normes IFRS, il existe une rubrique intermédiaire } \\
\text { " autres fonds propres » entre dettes et capitaux propres, dans } \\
\text { laquelle sont classés certains instruments financiers (titres } \\
\text { participatifs, titres subordonnées à durée indéterminé, obligations } \\
\text { remboursables en actions...). } \\
\text { Les actions propres constituent des actifs selon le SYSCOHADA et } \\
\text { ne viennent pas en diminution des capitaux propres comme en IFRS. }\end{array}$ & Divergence \\
\hline $\begin{array}{l}\text { IAS } 33 \\
\text { Résultat par action }\end{array}$ & $\begin{array}{l}\text { Au niveau du Plan Comptable Général : Traitement comptable non } \\
\text { développé dans le système comptable OHADA. Toutefois, le PCGO } \\
\text { demande de le fournir dans la Note } 31 \\
\text { Au niveau du dispositif relatif aux comptes consolidés et combinés : } \\
\text { Le dispositif prévoit de fournir au pied du compte de résultat } \\
\text { consolidé le résultat de base par action et le résultat dilué par action } \\
\text { conformément aux prescriptions de la norme IAS } 33\end{array}$ & Divergence \\
\hline $\begin{array}{c}\text { IAS 39 } \\
\text { Instruments financiers : } \\
\text { Comptabilisation et évaluation }\end{array}$ & $\begin{array}{l}\text { Il n'existe pas de classification formelle par catégories. Cependant, } \\
\text { selon les IFRS les actifs financiers sont classés selon quatre } \\
\text { catégories et les passifs } \\
\text { Selon le Système Comptable OHADA, le critère de classement d'un } \\
\text { instrument financier émis tient compte de la forme juridique. } \\
\text { Les passifs financiers sont classés soit en tant que passifs à la juste } \\
\text { valeur par le biais du compte de résultat, soit en autres passifs. } \\
\text { Créances et prêts évalués à leur valeur nominale et non selon le coût } \\
\text { amorti comme le précise les IFRS aux IFRS (taux d'intérêt effectif). }\end{array}$ & Divergence \\
\hline $\begin{array}{l}\text { IFRS 1 } \\
\text { Première application des normes } \\
\text { internationales d'information } \\
\text { financière }\end{array}$ & $\begin{array}{l}\text { Selon le Système comptable OHADA révisé, le passage du } \\
\text { SYSCOHADA actuel au SYSCOHADA révisé s'analyse comme un } \\
\text { changement de réglementation comptable mais avec un retraitement } \\
\text { rétrospectif par imputation de façon exceptionnelle et par dérogation } \\
\text { sur le compte } 475 \text { (au lieu d'une imputation sur les capitaux propres } \\
\text { selon les IFRS) }\end{array}$ & Divergence \\
\hline $\begin{array}{c}\text { IFRS } 2 \\
\text { Paiement fondé sur des actions }\end{array}$ & $\begin{array}{l}\text { Il n'y a pas d'évaluation en juste valeur des avantages consentis sous } \\
\text { forme de paiements en actions. Il existe toutefois des dispositions } \\
\text { spécifiques aux attributions d'actions gratuites. }\end{array}$ & Divergence \\
\hline
\end{tabular}




\begin{tabular}{|c|c|c|}
\hline $\begin{array}{l}\text { IFRS } 4 \\
\text { Contrat d'assurance }\end{array}$ & $\begin{array}{l}\text { Norme non retenue par le SYSCOHADA révisé (le code CIMA n'a } \\
\text { pas encore fait l'objet jusqu'à ce jour d'une convergence aux normes } \\
\text { IFRS). }\end{array}$ & Divergence \\
\hline $\begin{array}{l}\text { IFRS } 5 \\
\text { Actifs non courants détenus en } \\
\text { vue de la vente et activités } \\
\text { abandonnées }\end{array}$ & $\begin{array}{l}\text { Selon le SYSCOHADA révisé, dans les comptes individuels, il } \\
\text { n'existe pas de dispositions particulières pour les actifs non courants } \\
\text { (ou un groupe destiné à être cédé). Dans les comptes consolidés, il } \\
\text { existe une exemption de consolidation des filiales si et seulement si } \\
\text { elles ont été acquises uniquement en vue d'être cédées. }\end{array}$ & Divergence \\
\hline $\begin{array}{c}\text { IFRS 9 } \\
\text { Instruments financiers }\end{array}$ & Norme non retenue par le SYSCOHADA révisé. & Divergence \\
\hline $\begin{array}{c}\text { IFRS } 11 \\
\text { Partenariats }\end{array}$ & $\begin{array}{l}\text { La norme IFRS } 11 \text { distingue deux types de partenariats, selon les } \\
\text { droits et obligations des parties qui découlent de l'opération : les } \\
\text { activités conjointes et les coentreprises. Le dispositif comptable } \\
\text { relatif aux comptes consolidés et combinés ne prévoit pas de } \\
\text { distinction entre activités conjointes et coentreprise. }\end{array}$ & Divergence \\
\hline $\begin{array}{l}\text { IFRS 12 } \\
\text { Informations à fournir sur les } \\
\text { intérêts détenus dans d'autres } \\
\text { entités }\end{array}$ & $\begin{array}{l}\text { Cette norme n'a pas été retenue mais elle comporte certaines } \\
\text { dispositions similaires aux prescriptions relatives aux Notes annexes } \\
\text { des comptes consolidés du SYSCOHADA }\end{array}$ & Divergence \\
\hline $\begin{array}{c}\text { IFRS } 13 \\
\text { Évaluation de la juste valeur }\end{array}$ & $\begin{array}{l}\text { Les dispositions de cette norme n'ont pas été prises en compte par le } \\
\text { SYSCOHADA }\end{array}$ & Divergence \\
\hline $\begin{array}{c}\text { IFRS } \mathbf{1 4} \\
\text { Comptes de report réglementaires }\end{array}$ & $\begin{array}{l}\text { Les dispositions de cette norme n'ont pas été retenues par le } \\
\text { SYSCOHADA }\end{array}$ & Divergence \\
\hline
\end{tabular}

\section{Source : l'auteur}

Le tableau ci-dessus présente l'ensemble des points de divergences totale encore observé jusqu'à nos jours, entre le SYSCOHADA révisé et les normes IFRS. Toutefois, il existe pour certaines normes, des points de divergence partielle entre elles et le SYSCOHADA révisé. Ainsi, le tableau ci-dessous fait apparaitre l'ensemble de ces points de divergence partielle.

Tableau 2 : comparaison entre les normes comptables SYSCOHADA révisé et IFRS (points de divergences partielles)

\begin{tabular}{|c|l|c|}
\hline NORMES IAS/IFRS & \multicolumn{1}{|c|}{ SYSCOHADA Révisé } & Observations \\
\hline Cadre conceptuel & $\begin{array}{l}\text { L'information comptable produite selon le SYSCOHADA est en } \\
\text { direction de toutes les parties prenantes alors que celles de l'IASB est } \\
\text { orientées en priorité vers les investisseurs. }\end{array}$ & $\begin{array}{c}\text { Convergence } \\
\text { partielle }\end{array}$ \\
\hline $\begin{array}{c}\text { IAS 1 } \\
\text { Présentation des états }\end{array}$ & $\begin{array}{l}\text { Il n'existe pas d'état de variation des capitaux propres dans les } \\
\text { comptes sociaux du système comptable OHADA sauf dans les } \\
\text { Notes annexes des comptes consolidés. }\end{array}$ & $\begin{array}{c}\text { Convergence } \\
\text { partielle }\end{array}$ \\
\hline $\begin{array}{c}\text { IAS 8 } \\
\text { Méthodes comptables, } \\
\text { changements d'estimations } \\
\text { comptables et erreurs }\end{array}$ & $\begin{array}{l}\text { Le SYSCOHADA révisé a retenu les dispositions de la norme IAS 8 } \\
\text { à l'exception des points suivants en cas de changements de méthodes } \\
\text { : l'information comparative n'est pas retraitée mais présentée en pro- } \\
\text { forma dans les Notes annexes. } \\
\text { - Il est admis de comptabiliser l'application rétrospective des } \\
\text { changements de méthodes en charge pour des raisons juridiques et } \\
\text { fiscales }\end{array}$ & $\begin{array}{c}\text { Convergence } \\
\text { partielle }\end{array}$ \\
\hline
\end{tabular}




\begin{tabular}{|c|c|c|}
\hline $\begin{array}{l}\text { IAS 12 } \\
\text { Les Impôts sur le Résultat }\end{array}$ & $\begin{array}{l}\text { En matière d'impôt sur le résultat l'IASB distingue deux types de } \\
\text { résultats : impôts différés et impôts exigible. le SYSCOHADA ne } \\
\text { prend en compte que les impôts exigibles et les impôts différés sont } \\
\text { seulement pris en comptes dans les comptes consolidés. }\end{array}$ & $\begin{array}{l}\text { Convergence } \\
\text { partielle }\end{array}$ \\
\hline $\begin{array}{c}\text { IAS } 16 \\
\text { Immobilisations corporelles }\end{array}$ & $\begin{array}{l}\text { Pas de divergence majeure. Selon le Système Comptable OHADA : } \\
\text { - L'approche par composant est limitée à certains biens pour les } \\
\text { entités qui ne font pas appel public à l'épargne. Il n'y a pas de } \\
\text { possibilité de réévaluation isolée pour une classe d'actifs. }\end{array}$ & $\begin{array}{l}\text { Convergence } \\
\text { partielle }\end{array}$ \\
\hline $\begin{array}{c}\text { IAS } 17 \\
\text { Contrats de location }\end{array}$ & $\begin{array}{l}\text { Pas de divergence dans les principes. Mais le SYSCOHADA révisé a } \\
\text { choisi les dispositions de L'IFRS } 16 \text { (date publication : janvier 2016) }\end{array}$ & $\begin{array}{l}\text { Convergence } \\
\text { partielle }\end{array}$ \\
\hline $\begin{array}{l}\text { IAS } 19 \\
\text { Les avantages du } \\
\text { personnel }\end{array}$ & $\begin{array}{l}\text { Pas de divergence majeure. Contrairement aux l'IFRS, le Système } \\
\text { comptable OHADA limite l'obligation de l'évaluation des } \\
\text { indemnités de départ à la retraite par la méthode actuarielle aux } \\
\text { seules entités qui font appel public à l'épargne }\end{array}$ & $\begin{array}{l}\text { Convergence } \\
\text { partielle }\end{array}$ \\
\hline $\begin{array}{l}\text { IAS 24 } \\
\text { Information relative aux } \\
\text { parties liées }\end{array}$ & $\begin{array}{l}\text { Selon le système comptable OHADA, la notion de partie liée est plus } \\
\text { restrictive que dans les normes IFRS. }\end{array}$ & $\begin{array}{l}\text { Convergence } \\
\text { partielle }\end{array}$ \\
\hline $\begin{array}{l}\text { IAS } 27 \\
\text { états financiers individuels }\end{array}$ & $\begin{array}{l}\text { Le dispositif comptable relatif aux comptes consolidés et combinés } \\
\text { du SYSCOHADA préconise la comptabilisation au coût des } \\
\text { participations dans les états financiers individuels de l'entité mère } \\
\text { conformément aux dispositions du Plan Comptable Général OHADA } \\
\text { (PCGO). } \\
\text { Selon les normes IFRS les participations dans des filiales, des } \\
\text { entreprises associées et des coentreprises (autres que celles classées } \\
\text { comme détenues en vue de la vente selon IFRS 5) sont } \\
\text { comptabilisées soit au coût, soit en tant qu'investissement selon IFRS } \\
9 \text { ou IAS } 39 \text {. }\end{array}$ & $\begin{array}{l}\text { Convergence } \\
\text { partielle }\end{array}$ \\
\hline $\begin{array}{l}\text { IAS 34 } \\
\text { Information financière } \\
\text { intermédiaire }\end{array}$ & $\begin{array}{l}\text { Les dispositions sont globalement similaires, avec des divergences en } \\
\text { termes de données comparatives }\end{array}$ & $\begin{array}{l}\text { Convergence } \\
\text { partielle }\end{array}$ \\
\hline $\begin{array}{c}\text { IAS 36 } \\
\text { Dépréciation d'actifs }\end{array}$ & $\begin{array}{l}\text { Selon le SYSCOHADA, une perte de valeur est comptabilisée } \\
\text { lorsque la valeur actuelle d'un actif est inférieure à sa valeur nette } \\
\text { comptable. } \\
\text { La valeur recouvrable est donc la valeur qui correspond au coût } \\
\text { actuel de l'actif et non la valeur la plus élevée entre sa juste valeur } \\
\text { diminuée des coûts de la sortie et sa valeur d'utilité. }\end{array}$ & $\begin{array}{l}\text { Convergence } \\
\text { partielle }\end{array}$ \\
\hline $\begin{array}{c}\text { IAS } 37 \\
\text { Provisions, passifs éventuels } \\
\text { et actifs éventuels }\end{array}$ & $\begin{array}{l}\text { D'une manière générale les dispositions de la norme IAS } 37 \text { ont été } \\
\text { retenues par le SYSCOHADA. Toutefois, il existe certaines } \\
\text { divergences qui portent sur les points suivants: } \\
\square \text { Le SYSCOHADA admet de façon dérogatoire les provisions } \\
\text { réglementées qui ne répondent pas en principe à la définition d'une } \\
\text { provision. } \\
\square \text { Les capitaux propres sont des passifs internes alors qu'en IFRS } \\
\text { seules les provisions et des dettes envers les tiers constituent des } \\
\text { passifs. }\end{array}$ & $\begin{array}{l}\text { Convergence } \\
\text { partielle }\end{array}$ \\
\hline $\begin{array}{c}\text { IAS } 38 \\
\text { Immobilisations incorporelles }\end{array}$ & $\begin{array}{l}\text { D'une manière générale les dispositions de la norme IAS } 38 \text { ont été } \\
\text { retenues par le SYSCOHADA. Toutefois, il existe certaines } \\
\text { divergences qui portent sur les points suivants : } \\
\square \text { Le fonds commercial est déprécié que lorsqu'il existe un indice de } \\
\text { perte de valeur. } \\
\square \text { Selon le SYSCOHADA, il n'y a pas de réévaluation possible des } \\
\text { immobilisations incorporelles. }\end{array}$ & $\begin{array}{l}\text { Convergence } \\
\text { partielle }\end{array}$ \\
\hline $\begin{array}{c}\text { IAS } 40 \\
\text { Les immeubles de placement }\end{array}$ & $\begin{array}{l}\text { Les dispositions de la norme IAS } 40 \text { ont été retenues par le } \\
\text { SYSCOHADA. Toutefois, après la comptabilisation initiale, tout }\end{array}$ & $\begin{array}{c}\text { Convergence } \\
\text { partielle }\end{array}$ \\
\hline
\end{tabular}




\begin{tabular}{|c|c|c|}
\hline & $\begin{array}{l}\text { immeuble de placement doit être évalué uniquement selon le modèle } \\
\text { du coût contrairement aux normes IFRS qui admet aussi l'évaluation } \\
\text { selon le modèle de la juste valeur. }\end{array}$ & \\
\hline $\begin{array}{l}\text { IAS } 41 \\
\text { Agriculture }\end{array}$ & $\begin{array}{l}\text { Les dispositions de la norme IAS } 41 \text { ont été retenues par le } \\
\text { SYSCOHADA. Toutefois, un actif biologique ou une production } \\
\text { agricole doit être comptabilisé selon le modèle du coût. }\end{array}$ & $\begin{array}{l}\text { Convergence } \\
\text { partielle }\end{array}$ \\
\hline $\begin{array}{c}\text { IFRS 3 } \\
\text { Regroupements d'entreprises }\end{array}$ & $\begin{array}{l}\text { Selon le dispositif comptable relatif aux comptes consolidés et } \\
\text { combinés, seule la méthode du goodwill partiel est autorisée. La } \\
\text { méthode du full goodwill n'est pas autorisée (les IFRS admettent les } \\
\text { deux méthodes). }\end{array}$ & $\begin{array}{l}\text { Convergence } \\
\text { partielle }\end{array}$ \\
\hline $\begin{array}{c}\text { IFRS } 10 \\
\text { états financiers consolidés }\end{array}$ & $\begin{array}{l}\text { Selon le SYSCOHADA, le contrôle se définit comme le pouvoir de } \\
\text { diriger les politiques financières et opérationnelles d'une entité afin } \\
\text { de tirer avantage de ses activités. } \\
\text { La notion de contrôle de fait est différente. En IFRS, il n'existe } \\
\text { aucune présomption, le contrôle doit être fait au cas par cas. }\end{array}$ & $\begin{array}{l}\text { Convergence } \\
\text { partielle }\end{array}$ \\
\hline $\begin{array}{c}\text { IFRS 15 } \\
\text { Produits des activités } \\
\text { ordinaires tirés de contrats } \\
\text { conclus avec des clients }\end{array}$ & $\begin{array}{l}\text { Les dispositions relatives à l'activation du coût d'obtention d'un } \\
\text { contrat ont été retenues par le SYSCOHADA. }\end{array}$ & $\begin{array}{l}\text { Convergence } \\
\text { partielle }\end{array}$ \\
\hline $\begin{array}{l}\text { IFRS } 16 \\
\text { Contrats de location }\end{array}$ & $\begin{array}{l}\text { Pas de divergence majeure mais le SYSCOHADA limite les } \\
\text { dispositions relatives au contrat de location chez le preneur au contrat } \\
\text { de crédit-bail, au contrat de location-vente et à tout autre contrat de } \\
\text { location ayant une option d'achat exerçable. Par contre aucun seuil } \\
\text { de signification n'a été fixé pour les locations de faible valeur. }\end{array}$ & $\begin{array}{l}\text { Convergence } \\
\text { partielle }\end{array}$ \\
\hline
\end{tabular}

Source : l'auteur, inspiré de l'AUDCIF et du guide d'application des IFRS

\section{Adoption des normes IFRS par l'OHADA : un choix aux enjeux multiples}

On peut conclure à première vue que le référentiel comptable IFRS réussit à réaliser l'objectif initialement définit par l'IASB au regard de la comparaison faite entre les principes fondamentaux sur lesquels se basent le référentiel OHADA et celui de l'IASB. Ce constat est tributaire de la traduction réelle et fidèle de la situation économique de toute entreprise qui adopte les normes IFRS. Ces dernières (normes IFRS) ont l'avantage de mettre en premier plan, la situation économique des entreprises, tandis que les normes locales ont une vision orientée vers le patrimoine. Dès lors, l'application des normes IFRS améliore la présentation de l'information financière inhérente au marché boursier et réduire de ce fait les problèmes de son efficience.

Bien que l'adoption de telles normes parait bénéfique, elle est porteuse de multiples enjeux auxquels doivent faire face les entreprises de la zone OHADA. Il s'agit entre autre, de parler un langage commun des affaires, de pouvoir lever les capitaux sur les place financières internationale et surtout de faire face à la concurrence acharnée. De ce fait, ces entreprises doivent être considérablement sensibilisées et orientées au risque d'être en marge de l'évolution internationale qui aujourd'hui est irréversibles et donc seules les limites restent inconnues. Ce veux a été évoqué dans la conclusion de Feudjo (2010). Pour cet auteur, la convergence des normes OHADA vers les normes internationales 
est une exigence urgente au risque de voire les entreprises de cet espace être marginalisées par les grands groupes européens.

\section{Conclusion}

L'adoption des normes IFRS par l'OHADA constitue un vrai défit, bien que ces normes permettent aux entreprises adoptives d'avoir plus de visibilité sur les marchés internationaux. Toutefois, ce choix considéré comme une révolution comptable, exige la mobilisation des ressources importantes. Malgré les changements et implications de nature comptables, l'adoption des normes IFRS exige une forte mobilisation des moyens techniques, humains et financiers, tant au niveau de l'instance de normalisation qu'au niveau des entreprises elle mêmes.

L'introduction des innovations comptables et l'amélioration de certains traitements est un enjeu majeur, principalement pour les entreprises structurellement plus petite et ne disposant pas les mêmes ressource internes que celle des grandes entreprises cotées. Cependant, la complexité normative et technique des normes IFRS a mis au devant de la scène, les limites des praticiens ne leur permettant pas d'appréhender l'intégralité du corpus de façon optimale.

Toutefois, plusieurs études réalisées jusqu'ici (Feudjo et al. 2021 ; Nyasseu, 2021) montrent que les entreprises de l'espace OHADA sont favorables aux normes IFRS, étant donnée qu'elles s'inscrivent dans la suite logique de la mondialisation des marchés. Ces résultats rejoignent ceux de Yepdo (2021) mené auprès des analystes financiers et de Ninyum (2021) auprès des experts comptables. Cependant, ces différents acteurs martèlent que la prise en compte du contraste contextuel semble être ignorée par le normalisateur dans ce processus. Ce changement de philosophie comptable à travers l'adoption des normes IFRS par les entreprises de la zone OHADA nous pousse à nous interroger sur la pertinence des informations comptables et financières produites, comparativement à celles produites selon les normes OHADA.

Loin d'être un simple outil de présentation et de communication de l'information financière, les IFRS constituent pour les entreprises de la zone OHADA, un levier d'attraction des investisseurs et d'intégration économique. De même, l'adoption de ces normes par les entreprises de cet espace s'apparente à un écu érigé face au vent violent de la mondialisation.

\section{Bibliographie}

[1] Anne le manh-bena, (2009). Le processus de normalisation comptable par l'IASB : le cas du résultat. Thèse de doctorat

[2] Barbu, E. (2006). L'application des normes IAS/IFRS par les entreprises françaises cotées : une décision sous influence institutionnelle, $27^{\text {ème }}$ Congrès de l'AFC Tunis. 
[3] Barbu, E. et Piot C. (2012). L'adoption des IAS / IFRS par les groupes français cotés. Volonté ou contrainte ?, Revue Française de Gestion, n²26, p. 53-74, 2012.

[4] Barbu, E. (2004). L'harmonisation comptable internationale : d'un vagabondage comptable à l'autre, Comptabilité - Contrôle - Audit 2004/1 (Tome 10), p. 37-61.DOI $10.3917 /$ cca.101.0037

[5] Cormier, D. et al. (2007). Le référentiel IFRS : nous dirigeons-nous vers une comptabilité audelà du réel ?, Comptabilité - Contrôle - Audit / Numéro thématique, p. 43-56, décembre 2007.

[6] Dafir, A. (2013). "La diplomatie économique marocaine en Afrique subsaharienne : réalités et enjeux, "Géoéconomie, n 4, p. 73-83, 2013.

[7] Danjou, P. (2013). Une mise au point concernant les International Financial Reporting Standards, news D.F.C.G, VOX-FI, février.

[8] Démaria, S. (2008). “Changement de normes : la stabilité des choix comptables," $29^{\text {eme }}$ Congrès de l'AFC, 29 et 30 mai 2008, ESSEC Cergy Pontoise, 2008.

[9] Dimaggio, P. et Powell W. (1983). The iron cage revisited: institutional isomorphism and collective rationality in organizational fields, American sociological review, Vol.48, $\mathrm{n}^{\circ} 2$, pp.147-160.

[10] Disle, C. et Noël C. (2007). La révolution des normes IFRS : une convergence de la comptabilité vers la finance ?, La Revue des Sciences de Gestion, Direction et Gestion, n42, pp.17-27.

[11] Elatife, H. (2012). Passage aux normes comptables internationales IAS/IFRS : Essai d'observation et de compréhension des choix effectués par les sociétés cotées à la Bourse des Valeurs de Casablanca, Thèse de doctorat, 2011-2012.

[12] Feudjo, J.R. Kaotoing S. Kouam T.E.E. et Kueda W.B. (2021). Pertinence des IFRS telle que perçue par les professionnels de la comptabilité dans les pays en voie de développement la revue du financier, Vol. $\mathrm{n}^{\circ}$, pp.61-79

[13] Feudjo, J.R. (2010). Harmonisation des normes africaines (OHADA) et internationales (IAS/IFRS) : une urgence ou une exigence ? , La Revue des Sciences de Gestion 2010/5 (n²45246), p. 149-158. DOI 10.3917/rsg.245.0149. Financière, p. 23-35.

[14] Haoudi, K. (2015). "Passage aux normes IFRS au Maroc : fondements théoriques, intérêt et enjeux » International Journal of Innovation and Applied Studies ISSN 2028-9324 Vol. 10 No. 4 Mar. 2015, pp. 1299-1311

[15] Mezias, S. (1990). An Institutional Model of Organizational Practice: Financial Reporting at the Fortune 200, Administrative science quarterly, Vol.15, n¹990, pp.431-457. 
[16] Ninyum, P.A. (2021). Arrimage du SYSCOHADA aux normes IFRS: une appréciation des experts comptables. Thèse de doctorat Ph.D, Université de Dschang, Cameroun, 350p.

[17] Nyasseu, E. J. (2021). Perception de l'évolution des normes OHADA vers les normes IFRS par les dirigeants d'entreprises membres du GICAM. Thèse de doctorat Ph.D, Université de Dschang, Cameroun, 385p.

[18] Touron, P. (2000). Apports et Limites de la théorie institutionnelle des organisations, Étude de trois cas d'adoption de normes comptables internationales en France, Actes du $21^{\text {ème }}$ congrès de l'AFC, Angers

[19] Waha, J. P. (2013). Proposition de convergence du référentiel comptable OHADA vers les normes IFRS : cas des immobilisations et autres dispositions essentielles. Mémoire d'expertise comptable, Cameroun.

[20] Yepdo, F.G. (2021). Pertinence de la convergence des normes OHADA vers les IFRS. Thèse de doctorat Ph.D, Université de Dschang, Cameroun, 477p. 